\title{
MC-CDMA Aided Multi-User Space-Time Shift Keying in Wideband Channels
}

\author{
Mohammad Ismat Kadir*, Shinya Sugiura ${ }^{\dagger}$, Sheng Chen* and Lajos Hanzo* \\ ${ }^{*}$ School of ECS, University of Southampton, SO17 1BJ, United Kingdom. \\ Email: \{mik1g09,sqc,lh\}@ecs.soton.ac.uk, http://www-mobile.ecs.soton.ac.uk \\ †Toyota Central R\&D Labs., Inc., Aichi, 480-1192, Japan. Email: sugiura@ieee.org
}

\begin{abstract}
In this paper, we propose multi-carrier code division multiple access (MC-CDMA)-aided space-time shift keying (STSK) for mitigating the performance erosion of the classic STSK scheme in dispersive channels, while supporting multiple users. The codewords generated by the STSK scheme are appropriately spread in frequency-domain (FD) and transmitted over a number of parallel frequency-flat subchannels. We propose a new receiver architecture amalgamating the single-stream maximumlikelihood (ML) detector of the STSK system and the multiuser detector (MUD) of the MC-CDMA system. The performance of the proposed scheme is evaluated for transmission over frequency-selective channels in both uncoded and channel-coded scenarios. The results of our simulations demonstrate that the proposed scheme overcomes the channel impairments imposed by wideband channels and exhibits near-capacity performance in a channel-coded scenario.
\end{abstract}

\section{INTRODUCTION}

Multiple-input multiple-output (MIMO) wireless systems have attracted substantial research interests over the past two decades owing to their potential of attaining beneficial diversity and/or multiplexing gains. The pioneering study of Foschini [1] reveals that MIMO systems have the capability to achieve a high transmission rate without requiring additional bandwidth. On the other hand, space-time block codes (STBCs) [2] were designed for improving the link reliability by the maximum attainable spatial diversity.

It is, however, not desirable that antenna elements (AEs) will be used either entirely for multiplexing or solely for diversity. Specifically, a particular MIMO configuration may be utilized for attaining both the diversity and the multiplexing gains. To this end, linear dispersion codes were proposed [3], [4], which outperformed the previous systems, but the decoding complexity was substantially increased. As a low-complexity design alternative, spatial modulation (SM) [5] as well as space shift keying (SSK) [6] adopted the principle of shrewd transmit-antenna activation to provide additional bandwidth efficiency. Motivated by these ideas, STSK [7], [8] was proposed, which extends the concept of pure spatial-domain antenna activation of SM/SSK schemes to both the spatial and temporal dimensions. To be more specific, the idea is to rely on beneficial dispersion matrix (DM) activation, rather than on the simple antenna activation process of SM/SSK in addition to the conventional modulation based signalling. The STSK system can thus provide substantial diversity as well as multiplexing gain. However, the majority of STSK studies were focused on narrowband scenarios, rather than on realistic wideband scenarios. Although the mitigation of the impairments due to channel dispersion was studied in [9], [10], the spreading of user information across the FD remained an open problem.

Against this background, our main contributions are:

1) We propose MC-CDMA aided STSK systems, which can attain a beneficial diversity versus multiplexing gain tradeoff even in multi-path environments, while supporting multi-user transmissions. The space-time codewords generated by STSK are appropriately mapped to the MC-CDMA subcarriers. As a result, the STSK signal generated for each subcarrier of the parallel modem experiences frequency-flat fading. FD spreading provides additional diversity benefits.

2) We propose a novel MUD amalgamated with the lowcomplexity single-stream ML detector of [7], [8] in order to estimate the user information which is spread over different subcarriers. We consider both the multiuser downlink (DL) and uplink (UL) scenarios and evaluate their performances against both the single-user and narrowband benchmarkers.

3) Furthermore, we design a near-capacity coding assisted MC-CDMA STSK arrangement and evaluate its performance.

The rest of the paper is organized as follows. Section II details the transceiver model of our MC-CDMA aided STSK system. The channel-coded MC-CDMA aided STSK philosophy is discussed in Section III. In Section IV, the system performance is characterized for both the uncoded and channelcoded scenarios. Finally, we conclude in Section V.

\section{SySTEM MODEL}

Consider an MC-CDMA aided STSK system having $N_{T}$ transmit and $N_{R}$ receive AEs and communicating over frequency-selective Rayleigh fading channels. Furthermore, $N_{c}$ subcarriers are employed by our MC-CDMA modem for transmitting $N_{p}$ STSK codewords.

\section{A. The Transmitter and the Channel}

Fig. 1 depicts the transmitter model of our MC-CDMA aided STSK scheme. The STSK transmitter generates spacetime codewords from the users' source information. These codewords are further spread across the FD and are then mapped to a number of subcarriers, before being transmitted 


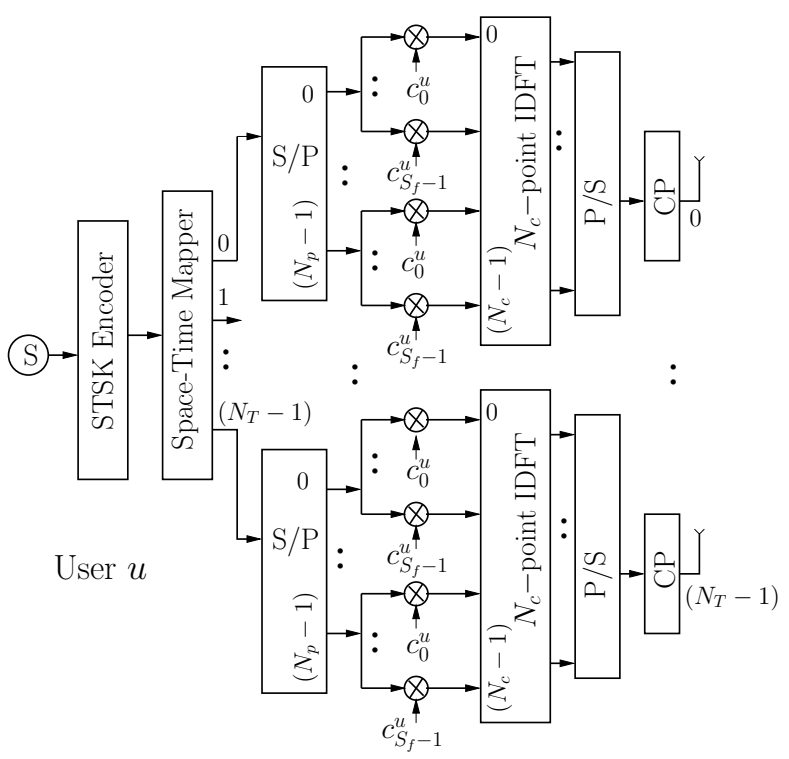

Fig. 1. Transmission model of the proposed MC-CDMA aided multiuser STSK scheme showing the transmission protocol for the generalized user $u$.

using $N_{T}$ transmit AEs over $T$ time slots. More specifically, each STSK signalling block $\boldsymbol{X}^{u}\left[n_{p}\right], n_{p}=0,1, \ldots,\left(N_{p}-1\right)$ is created from $\log _{2}(\mathcal{L} \cdot Q)$ source bits of user $u, u=$ $0,1, \ldots,(U-1)$, in accordance with [7], [8]

$$
\boldsymbol{X}^{u}\left[n_{p}\right]=x^{u}\left[n_{p}\right] \boldsymbol{M}^{u}\left[n_{p}\right],
$$

where $x^{u}\left[n_{p}\right]$ is an $\mathcal{L}$-ary modulation symbol mapped by $\log _{2} \mathcal{L}$ bits of $u$-th user's source information and $\boldsymbol{M}^{u}\left[n_{p}\right]$ is the DM activated from among $Q$ DMs $\left(M_{1}, M_{2}, \ldots, M_{Q}\right)$, determined by the remaining $\log _{2} Q$ bits. The DMs are appropriately optimized employing a certain objective function under the power constraint: $\operatorname{tr}\left(M_{q}^{H} M_{q}\right)=T \quad \forall q$, as detailed in [7], [8], [11]. The STSK scheme may be unambiguously described by the $\left(N_{T}, N_{R}, T, Q, \mathcal{L}\right)$ parameters.

The mapping of the STSK codewords to the $N_{c}$ subcarriers is illustrated in Fig. 2. A number of $N_{p}$ space-time codewords generated by the STSK encoder are spread across all the $N_{c}$ subcarriers by the user-specific spreading sequence, $\boldsymbol{C}^{u}=\left[c_{0}^{u}, c_{1}^{u}, \ldots, c_{S_{f}-1}^{u}\right]$, where $S_{f}$ represents the spreading factor and $u$ is the user index. To be more specific, assuming the relationship $N_{c}=\left(N_{p} \times S_{f}\right)$, FD spreading of the $N_{p}$ codeword symbols results in a number of $N_{c}$ FD symbols. The complex baseband representation of the MC-CDMA STSK time-domain (TD) signal of user $u$ transmitted in a particular signalling interval may thus be expressed as [12]-[14]:

$$
\begin{aligned}
\boldsymbol{s}_{n_{T}, T_{i}}^{u}(t)= & \frac{1}{\sqrt{N_{c}}} \sum_{n_{p}=0}^{\left(N_{p}-1\right)} \sum_{s_{f}=0}^{\left(S_{f}-1\right)} c_{s_{f}}^{u} \boldsymbol{X}_{n_{T}, T_{i}}^{u}\left[n_{p}\right] e^{j 2 \pi f_{n_{c}} t}, \\
& T_{i}=0,1, \ldots,(T-1) \quad 0<t<N_{c} \mathcal{T},
\end{aligned}
$$

where $(\bullet)_{n_{T}, T_{i}}^{u}$ denotes the $\left(n_{T}, T_{i}\right)$ element of the matrix $(\bullet)^{u}, \mathcal{T}$ represents the TD sampling interval, while $N_{c} \mathcal{T}$ is the TD frame interval. Furthermore, $f_{n_{c}}$ is the frequency of the $n_{c}$-th subcarrier and $n_{c}=\left(n_{p} S_{f}+s_{f}\right)$. In order to maintain
Transmit AE index, $n_{T} \quad$ Transmit AE index, $n_{T}$

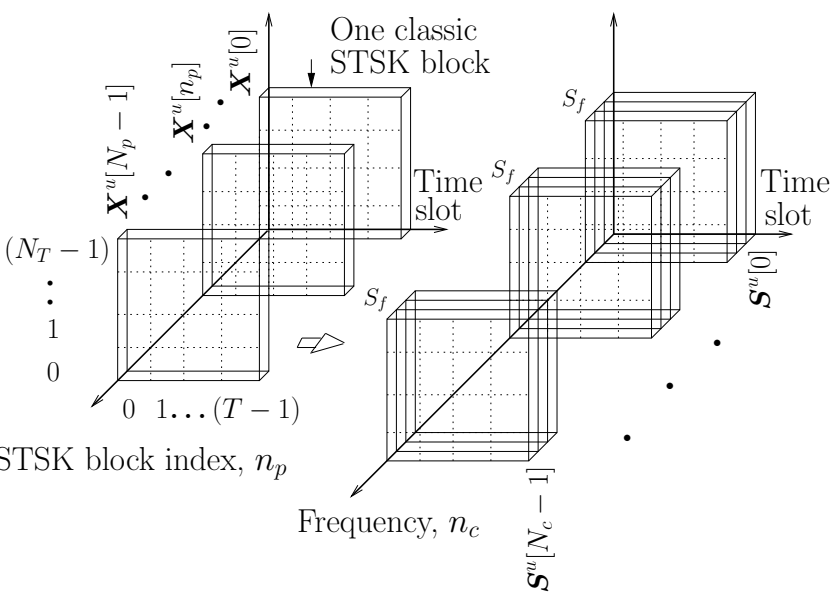

Fig. 2. Illustration of the mapping of $N_{p}$ STSK codewords to $N_{c}$ orthogonal sub-carriers. The STSK codewords are spread in FD by the user-specific $S_{f}$ chip spreading sequence and are then mapped to the sub-carriers.

orthogonality, the subcarrier frequencies have to be equally spaced with

$$
f_{n_{c}}=\frac{n_{c}}{N_{c} \mathcal{T}} .
$$

Defining the FD-spread symbol stream of user $u$ by $\boldsymbol{S}_{n_{T}, T_{i}}^{u}=c_{s_{f}}^{u} \boldsymbol{X}_{n_{T}, T_{i}}^{u}, \quad s_{f}=1,2, \ldots,\left(S_{f}-1\right)$, the transmit frame before and after FD spreading are

$$
\left[\boldsymbol{X}^{u}[0], \boldsymbol{X}^{u}[1], \ldots, \boldsymbol{X}^{u}\left[n_{p}\right], \ldots, \boldsymbol{X}^{u}\left[N_{p}-1\right]\right]
$$

and

$$
\left[\boldsymbol{S}^{u}[0], \boldsymbol{S}^{u}[1], \ldots, \boldsymbol{S}^{u}\left[n_{c}\right], \ldots, \boldsymbol{S}^{u}\left[N_{c}-1\right]\right],
$$

respectively. The MC-CDMA TD samples may thus be expressed by

$$
\begin{array}{r}
\boldsymbol{s}_{n_{T}, T_{i}}^{u}\left[n_{s}\right]=\frac{1}{\sqrt{N_{c}}} \sum_{n_{c}=0}^{\left(N_{c}-1\right)} \boldsymbol{S}_{n_{T}, T_{i}}^{u}\left[n_{c}\right] e^{j 2 \pi \frac{n_{s} n_{c}}{N_{c}}}, \\
0 \leq n_{s} \leq\left(N_{c}-1\right),
\end{array}
$$

which is given by the $N_{c}$-point inverse discrete Fourier transform (IDFT) of $\boldsymbol{S}_{n_{T}, T_{i}}^{u}$ :

$$
\boldsymbol{s}_{n_{T}, T_{i}}^{u}=\operatorname{IDFT}_{N_{c}}\left\{\boldsymbol{S}_{n_{T}, T_{i}}^{u}\right\} .
$$

After the IDFT operation, cyclic prefices (CP) of appropriate length are incorporated for eliminating the effects of intersymbol interference (ISI).

We assume that each channel component of the MIMO system corresponding to user $u$ is frequency-selective, and is described by its discrete-time channel impulse response (CIR), $\boldsymbol{h}_{n_{R}, n_{T}}^{u}, \quad n_{R}=0,1, \ldots,\left(N_{R}-1\right), \quad n_{T}=0,1, \ldots,\left(N_{T}-\right.$ $1)$, whereas $\tilde{\boldsymbol{H}}^{u}$ denotes the corresponding $\left(N_{R} \times N_{T}\right)$ element FD channel transfer matrix.

1) Multi-user uplink (UL) scenario: In the multi-user UL scenario, the signals from the mobile stations (MSs) of different users are received at the base station's (BS) AEs through different channels. Hence the TD and the FD channel coefficients are independent between users. 
2) Multi-user downlink (DL) scenario: During DL transmissions, the desired signal and the interferring signals are received at any MS through the same channel. The channel variables for all the users may thus be expressed as identical to those of the intended ${ }^{1}$ user $v$ for DL transmissions:

$$
\boldsymbol{h}_{n_{R}, n_{T}}^{u}=\boldsymbol{h}_{n_{R}, n_{T}}^{v} \quad \tilde{\boldsymbol{H}}^{u}=\tilde{\boldsymbol{H}}^{v} \quad \forall u .
$$

\section{B. The Receiver}

Fig. 3 illustrates the receiver architecture of our MCCDMA aided STSK system. The received signal, after CP removal, is demodulated by Fourier-transforming it. Assuming perfect synchronization at the receiver, the discrete-time signal impinging on the $n_{R}$-th receive $\mathrm{AE}$ during time interval $T_{i}$ can be expressed as [14]

$$
\boldsymbol{r}_{n_{R}, T_{i}}=\sum_{u=0}^{(U-1)} \sum_{n_{T}=0}^{\left(N_{T}-1\right)} \boldsymbol{h}_{n_{R}, n_{T}}^{u} \circledast \boldsymbol{s}_{n_{T}, T_{i}}^{u}+\boldsymbol{w}_{n_{R}, T_{i}},
$$

where $\circledast$ denotes $N_{c}$-point circular convolution and $\boldsymbol{w}_{n_{R}, T_{i}}$ represents the additive white Gaussian noise (AWGN). After applying $N_{c}$-point discrete Fourier transform (DFT) denoted by $\operatorname{DFT}_{N_{c}}\{\cdot\}$, the FD MIMO output $\boldsymbol{R}\left[n_{c}\right]$ is given by

$$
\begin{array}{r}
\boldsymbol{R}\left[n_{c}\right]=\sum_{u=0}^{(U-1)} \tilde{\boldsymbol{H}}^{u}\left[n_{c}\right] \boldsymbol{S}^{u}\left[n_{c}\right]+\boldsymbol{W}\left[n_{c}\right] \\
=\underbrace{\tilde{\boldsymbol{H}}^{v}\left[n_{c}\right] \boldsymbol{S}^{v}\left[n_{c}\right]}_{\text {contribution from desired signal }}+\underbrace{\sum_{\substack{u=0 \\
u \neq v}}^{(U-1)} \tilde{\boldsymbol{H}}^{u}\left[n_{c}\right] \boldsymbol{S}^{u}\left[n_{c}\right]}_{\text {additive noise }} \\
+\underbrace{\boldsymbol{W}\left[n_{c}\right]}_{\text {multi-user interference }}
\end{array}
$$

for every $n_{c}=0,1, \ldots,\left(N_{c}-1\right)$, such that

$$
\begin{aligned}
\boldsymbol{R}_{n_{R}, T_{i}}=\operatorname{DFT}_{N_{c}}\left\{\boldsymbol{r}_{n_{R}, T_{i}}\right\} & \boldsymbol{R}\left[n_{c}\right] \in \mathbb{C}^{N_{R} \times T}, \\
\tilde{\boldsymbol{H}}_{n_{R}, n_{T}}^{u}=\operatorname{DFT}_{N_{c}}\left\{\boldsymbol{h}_{n_{R}, n_{T}}^{u}\right\} & \tilde{\boldsymbol{H}}^{u}\left[n_{c}\right] \in \mathbb{C}^{N_{R} \times N_{T}} \\
\boldsymbol{S}_{n_{T}, T_{i}}^{u}=\operatorname{DFT}_{N_{c}}\left\{\boldsymbol{s}_{n_{T}, T_{i}}^{u}\right\} & \boldsymbol{S}^{u}\left[n_{c}\right] \in \mathbb{C}^{N_{T} \times T}, \\
\boldsymbol{W}_{n_{R}, T_{i}}=\operatorname{DFT}_{N_{c}}\left\{\boldsymbol{w}_{n_{R}, T_{i}}\right\} & \boldsymbol{W}\left[n_{c}\right] \in \mathbb{C}^{N_{R} \times T}
\end{aligned}
$$

The linearized system model of [4] reduces (8) to

$$
\begin{aligned}
\overline{\boldsymbol{R}}\left[n_{c}\right] & =\sum_{u=0}^{(U-1)} \tilde{\boldsymbol{H}}^{u}\left[n_{c}\right] \boldsymbol{\chi}\left[c_{s_{f}}^{u} \boldsymbol{K}^{u}\right]+\overline{\boldsymbol{W}}\left[n_{c}\right], \\
s_{f} & =1,2, \ldots,\left(S_{f}-1\right) \quad n_{c}=\left(n_{p} S_{f}+s_{f}\right),
\end{aligned}
$$

where we have $\overline{\boldsymbol{R}}\left[n_{c}\right]=\operatorname{vec}\left(\boldsymbol{R}\left[n_{c}\right]\right) \in \mathbb{C}^{N_{R} T \times 1}$ by using the vectorial stacking operator $\operatorname{vec}(\cdot), \tilde{\boldsymbol{H}}^{u}\left[n_{c}\right]=\boldsymbol{I}_{T} \otimes$ $\tilde{\boldsymbol{H}}^{u}\left[n_{c}\right] \in \mathbb{C}^{N_{R} T \times N_{T} T}$ is the stacked FD channel transfer matrix of user $u, \otimes$ denotes the Kronecker product and $\boldsymbol{I}_{T}$ represents the $(T \times T)$ identity matrix, and the linear transformation matrix $\chi \in \mathbb{C}^{N_{T} T \times Q}$ [4], [8] is given by $\chi=\left[\operatorname{vec}\left(\boldsymbol{M}_{1}\right), \ldots, \operatorname{vec}\left(\boldsymbol{M}_{Q}\right)\right]$. Furthermore, $\overline{\boldsymbol{W}}\left[n_{c}\right]=$

\footnotetext{
${ }^{1}$ We use the notation $u$ to represent the generalized user and $v$ to denote the intended user.
}

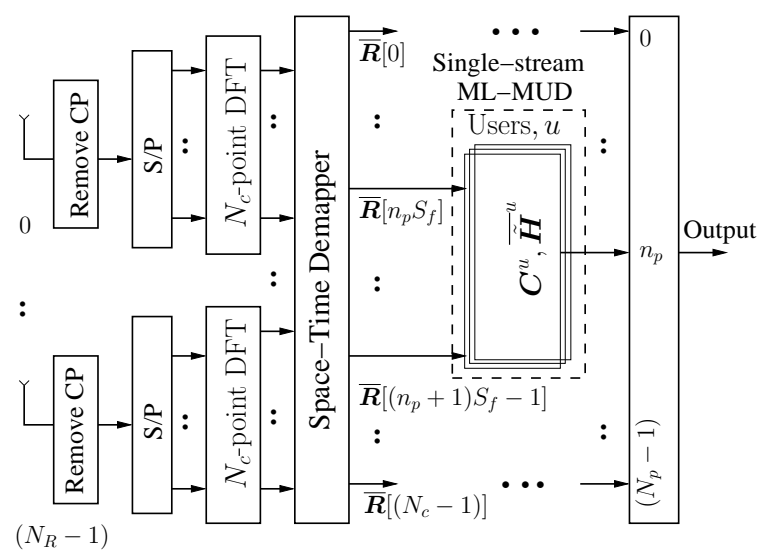

Fig. 3. Receiver architecture of the proposed multi-user MC-CDMA aided STSK scheme.

$\operatorname{vec}\left(\boldsymbol{W}\left[n_{c}\right]\right) \in \mathbb{C}^{N_{R} T \times 1}$ is the $\mathrm{AWGN}$ vector, while the equivalent transmit signal vector $\boldsymbol{K}^{u} \in \mathbb{C}^{Q \times 1}$ is defined by [8]

$$
\boldsymbol{K}^{u}=[\underbrace{0, \ldots, 0}_{q-1}, x^{u}, \underbrace{0, \ldots, 0}_{Q-q}]^{T}
$$

where a constellation symbol $x^{u}$ exists only at position $q$, such that the $q$-th DM is activated.

Since the source information of users in a particular spacetime block indexed by $n_{p}$ is spread over $S_{f}$ number of outputs from $\overline{\boldsymbol{R}}\left[n_{p} S_{f}\right]$ to $\overline{\boldsymbol{R}}\left[\left(n_{p}+1\right) S_{f}-1\right]$, the single-stream maximum-likelihood (ML) detector of [7], [8] in combination with the multi-user detector (MUD) of [12] is employed over these $S_{f}$ number of matrices to jointly detect the information of the users corresponding to the specific block. The single-stream maximum-likelihood multiuser detector (MLMUD) [7], [12] may hence be used for estimating the set of DM indices, $\boldsymbol{q}\left[n_{p}\right]=\left\{q^{0}\left[n_{p}\right], \ldots, q^{(U-1)}\left[n_{p}\right]\right\}$ and the constellation symbol indices, $\boldsymbol{l}_{c}\left[n_{p}\right]=\left\{l_{c}^{0}\left[n_{p}\right], \ldots, l_{c}^{(U-1)}\left[n_{p}\right]\right\}$. More explicitly, given the received signals of (14), the MLMUD may thus be formulated as ${ }^{2}$ [7], [12]:

$$
\begin{aligned}
\left(\hat{\boldsymbol{q}}\left[n_{p}\right], \hat{\boldsymbol{l}}_{c}\left[n_{p}\right]\right) & =\underset{\boldsymbol{q}, \boldsymbol{l}_{c}}{\arg \min } \sum_{s_{f}=0}^{\left(S_{f}-1\right)} \| \overline{\boldsymbol{R}}\left[n_{p} S_{f}+s_{f}\right] \\
& -\sum_{u=0}^{(U-1)} c_{s_{f}}^{u} \overline{\boldsymbol{H}}^{u}\left[n_{p} S_{f}+s_{f}\right] \boldsymbol{\chi} \boldsymbol{K}_{q^{u}, l_{c}^{u}}^{u} \|^{2} \\
& =\underset{\boldsymbol{q}, \boldsymbol{l}_{c}}{\arg \min } \sum_{s_{f}=0}^{\left(S_{f}-1\right)} \| \overline{\boldsymbol{R}}\left[n_{p} S_{f}+s_{f}\right] \\
& -\sum_{u=0}^{(U-1)} c_{s_{f}}^{u} s_{l_{c}^{u}}^{u}\left(\tilde{\boldsymbol{H}}^{u}\left[n_{p} S_{f}+s_{f}\right] \boldsymbol{\chi}\right)_{q^{u}} \|^{2},
\end{aligned}
$$

where $\boldsymbol{K}_{q^{u}, l_{c}^{u}}^{u}$ denotes the equivalent transmit signal vector defined in (15) when the transmitted indices are $q^{u}$ and $l_{c}^{u}$ respectively, $s_{l_{c}^{u}}^{u}$ denotes the $l_{c}^{u}$-th constellation symbol of user $u$ and $(\bullet)_{q^{u}}$ indicates the $q^{u}$-th column of the matrix ' $\bullet$ '.

${ }^{2}$ Equations (7) - (14) and (16) are applicable for both the UL and DL scenarios and may be simplified further using (6) for downlink channels. 


$$
L_{e}\left(b_{i}\right)=\ln \frac{\sum_{\boldsymbol{K}_{q^{u}, l_{c}^{u}}^{u} \in \boldsymbol{K}_{1}^{i}} \exp \left\{-\sum_{s_{f}=0}^{\left(S_{f}-1\right)}\left\|\overline{\boldsymbol{R}}\left[n_{p} S_{f}+s_{f}\right]-\sum_{u=0}^{(U-1)} c_{s_{f}}^{u} \overline{\tilde{\boldsymbol{H}}}^{u}\left[n_{p} S_{f}+s_{f}\right] \chi \boldsymbol{K}_{q^{u}, l_{c}^{u}}^{u}\right\|^{2} / N_{0}+\sum_{j \neq i} b_{j} L_{a}\left(b_{j}\right)\right\}}{\sum_{\boldsymbol{K}_{q^{u}, l_{c}^{u}}^{u} \in \boldsymbol{K}_{0}^{i}} \exp \left\{-\sum_{s_{f}=0}^{\left(S_{f}-1\right)}\left\|\overline{\boldsymbol{R}}\left[n_{p} S_{f}+s_{f}\right]-\sum_{u=0}^{(U-1)} c_{s_{f}}^{u} \overline{\tilde{\boldsymbol{H}}}^{u}\left[n_{p} S_{f}+s_{f}\right] \chi \boldsymbol{K}_{q^{u}, l_{c}^{u}}^{u}\right\|^{2} / N_{0}+\sum_{j \neq i} b_{j} L_{a}\left(b_{j}\right)\right\}}
$$

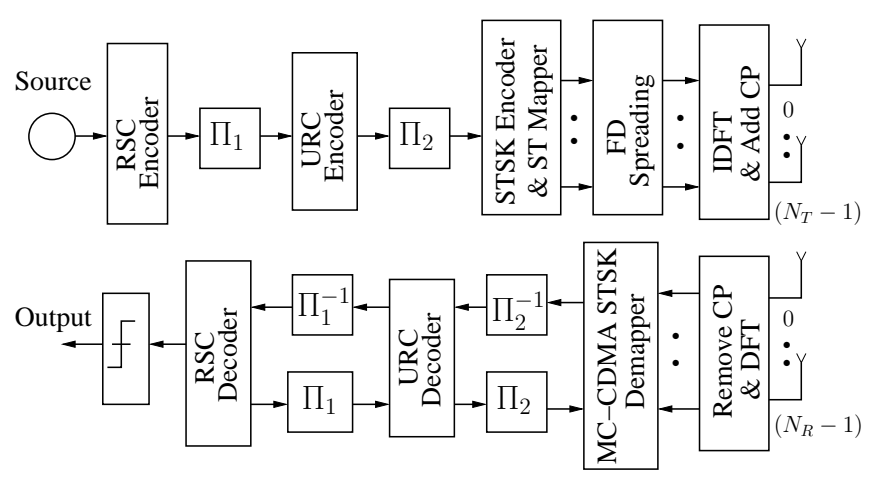

Fig. 4. The channel-coded MC-CDMA STSK scheme.

\section{Channel-Coded Scheme}

We propose a powerful iterative-detection aided MC-CDMA STSK transceiver as shown in Fig. 4. We employ a recursive systematic convolutional (RSC) and unity-rate coding (URC) architecture, where the information bits, after being channelencoded by the RSC code, are passed to the random bit interleaver $\Pi_{1}$. After being randomly permuted, these bits are then URC encoded ${ }^{3}$ and after a second interleaving by $\Pi_{2}$, are transmitted through the MC-CDMA STSK scheme.

The received signals, after discarding the $\mathrm{CP}$, are demodulated by the FD MC-CDMA demodulator. The symbols, after DFT processing at the demodulator, are input to the MC-CDMA STSK demapper block. Then, the three softdecision decoders (the STSK demapper, the URC and the RSC decoder) start exchanging extrinsic information iteratively. The URC decoder generates extrinsic information employing the a priori information gleaned from the STSK demapper. More specifically, if the band of linearized FD signals from $\overline{\boldsymbol{R}}\left[n_{p} S_{f}\right]$ to $\overline{\boldsymbol{R}}\left[\left(n_{p}+1\right) S_{f}-1\right]$ contains $N$ channel coded bits $b_{0}, b_{1}, \ldots, b_{(N-1)}$, then (17) written at the top of the page formulates the extrinsic logarithmic-likelihood ratio (LLR), $L_{e}\left(b_{i}\right)$ for the bit $b_{i}, i=0,1, \ldots,(N-1)$, as detailed in [11], [15]. In (17), $L_{a}(\bullet)$ refers to the a priori LLR for the bit ' $\bullet, \boldsymbol{K}_{1}^{i}$ and $\boldsymbol{K}_{0}^{i}$ denote the subsets of the possible $\boldsymbol{K}^{u}$ vectors defined by (15) corresponding to the bit values $b_{i}=1$ and $b_{i}=0$, respectively, whereas other notations were defined earlier in Sec. II. Equation (17) can be further simplified using the approximate-logarithmic-maximum a posteriori (Approx-

\footnotetext{
${ }^{3}$ The URC has been used to beneficially spread the extrinsic information owing to its infinite impulse response [11], thus facilitating iterative convergence to extremely low bit-error rate (BER).
}

Table I

MAIN SYSTEM PARAMETERS

\begin{tabular}{lr}
\hline Channel model & COST207-RA \\
Fast fading envelope & COST207-TU12 \\
Rormaleigh \\
Spreading code & 0.001 \\
No. of subcarriers, $N_{C}$ & Walsh-Hadamard \\
CP length & 64 \\
STSK $\left(N_{T}, N_{R}, T, Q, \mathcal{L}\right)$ & 16 \\
& $(2,2,2,2,2)$ \\
Outer RSC en/decoder & $(2,2,2,4,4)$ \\
& half rate \\
Generator polynomial & constraint length=2 \\
Interleaver length & $(011,010)_{2}$ \\
Outer iterations, $I_{\text {outer }}$ & $7,372,800$ bits \\
Inner en/decoder & 5 \\
Inner iterations, $I_{\text {inner }}$ & Unity Rate Code \\
& 2
\end{tabular}

$\log$-MAP) algorithm [16], yielding

$$
L_{e}\left(b_{i}\right)=\operatorname{jac}_{\boldsymbol{K}_{q^{u}, l_{c}^{u}}^{u} \in \boldsymbol{K}_{1}^{i}}(d)-\underset{\boldsymbol{K}_{q^{u}, l_{c}^{u}}^{u} \in \boldsymbol{K}_{0}^{i}}{\mathrm{jac}}(d),
$$

where $\underset{\boldsymbol{K}_{q^{u}, l_{c}^{u}}^{u} \in \boldsymbol{K}_{1}^{i}}{\mathrm{jac}}(d)$ and $\underset{\boldsymbol{K}_{q^{u}, l_{c}^{u}}^{u} \in \boldsymbol{K}_{0}^{i}}{\mathrm{jac}}(d)$ represent the Jacobian logarithm of the expression ' $d$ ', when $\boldsymbol{K}_{q^{u}, l_{c}^{u}}^{u}$ is a member of $\boldsymbol{K}_{1}^{i}$ and $\boldsymbol{K}_{0}^{i}$, respectively and where ' $d$ ' is given by:

$$
\begin{aligned}
d=- & \sum_{s_{f}=0}^{\left(S_{f}-1\right)} \| \overline{\boldsymbol{R}}\left[n_{p} S_{f}+s_{f}\right] \\
& \quad-\sum_{u=0}^{(U-1)} c_{s_{f}}^{u} \overline{\tilde{\boldsymbol{H}}}^{u}\left[n_{p} S_{f}+s_{f}\right] \chi \boldsymbol{K}_{q^{u}, l_{c}^{u}}^{u} \|^{2} / N_{0} \\
& +\sum_{j \neq i} b_{j} L_{a}\left(b_{j}\right) .
\end{aligned}
$$

The RSC decoder, after several iterative exchange of extrinsic information, outputs the estimate of the information bits.

\section{RESUlts AND Discussions}

We characterize STSK and MC-CDMA aided STSK scheme both in narrowband and wideband environments. The basic simulation parameters are listed in Table I.

It is demonstrated in Fig. 5 that classic single-carrier STSK $(2,2,2,2,2)$ scheme works well in narrowband scenarios, but it exhibits a severe error floor in dispersive channels, when the COST207-RA channel model is considered. In the typical 
This full-text paper was peer-reviewed and accepted to be presented at the IEEE WCNC 2013 conference.

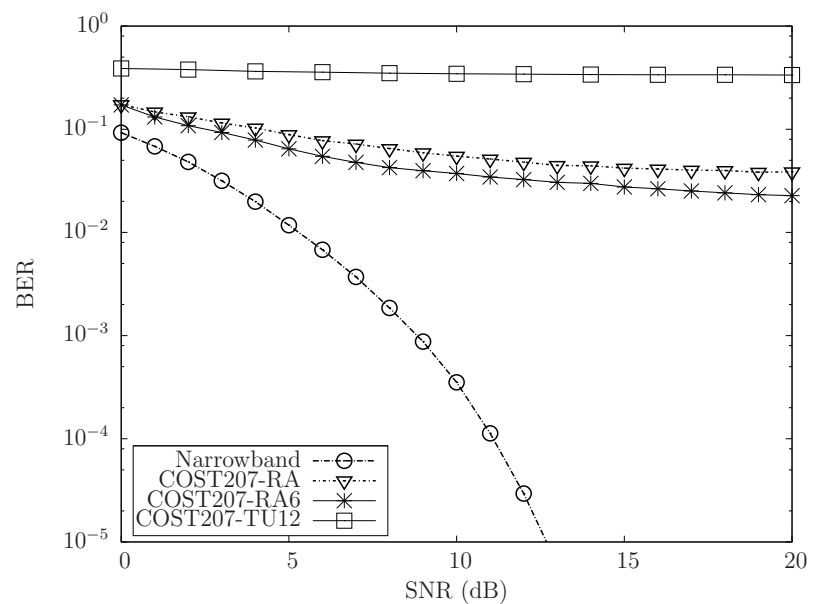

Fig. 5. BER performance of uncoded single-user classic STSK $\left(N_{T}, N_{R}, T, Q, \mathcal{L}\right)=(2,2,2,2,2)$ of [7], [8] under different channel models.

urban (TU) scenario, the classic STSK scheme suffers from a substantial further performance degradation.

By contrast, the effects of the above-mentioned channels on our MC-CDMA aided STSK scheme are illustrated by Fig. 6. As expected, both the STSK and MC-CDMA STSK schemes exhibits a similar performance as in narrowband channels owing to the absence of ISI. Furthermore, the MCCDMA STSK scheme is capable of maintaining its superior multiple antenna gain in both the rural area (RA) scenario modelled by the COST207-RA channel as well as in the TU scenario characterized by the COST207-TU12 model. Furthermore, the single-user MC-CDMA aided STSK scheme shows improved performance upon increasing $S_{f}$ both for the COST207-RA and COST207-TU12 channels owing to the fact that a FD spreading sequence may still remain recoverable, even when some of its chips are corrupted. The performance of the proposed MC-CDMA STSK $(2,2,2,2,2)$ and MC-CDMA $\operatorname{STSK}(2,2,2,4,4)$ in dispersive channels is also compared to that of the MC-CDMA based STBC $\left(N_{T}, N_{R}\right)=(2,2)$, BPSK and STBC $(2,2)$, QPSK respectively having similar throughput, which demonstrates the strength of the proposed scheme.

Additionally, the performances of the multi-user MCCDMA aided STSK scheme for the DL and the UL scenarios in the COST207-RA channel are characterized in Fig. 7 and Fig. 8 respectively, which were found to be more or less similar under the idealized conditions of perfect synchronization. Furthermore, the achievable performance improved upon increasing the $S_{f}$, while both the UL and the DL BER degraded under multiuser scenarios as a result of the increased MUI imposed by multiple users.

Fig. 9 characterizes the performance of the serially concatenated RSC- and URC-coded single-user MC-CDMA STSK $(2,2,2,4,4)$ scheme communicating over broadband channels. The investigation of our channel-coded scheme was carried out using the simulation parameters of Table I. Fig. 9 demonstrates that the channel-coded scheme provides a sharp decrease in

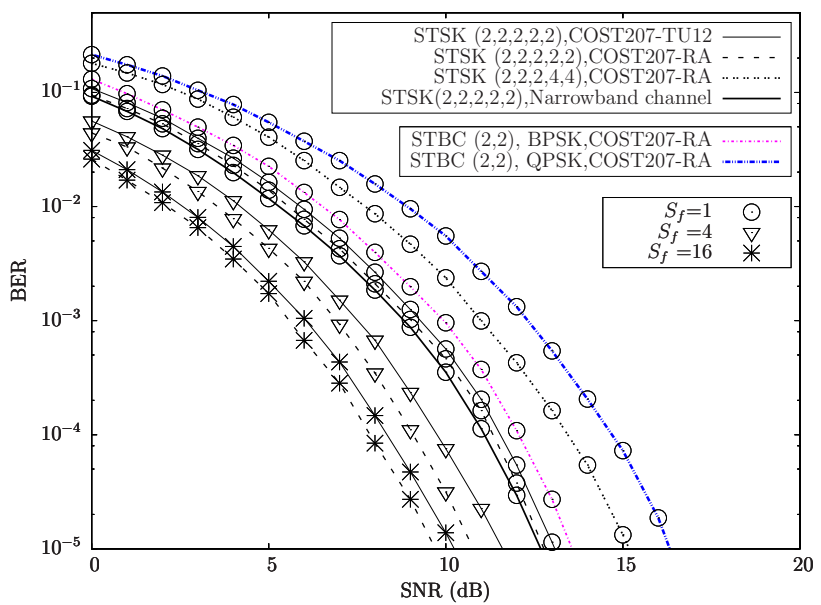

Fig. 6. Performance of single-user uncoded MC-CDMA STSK system both in narrowband and in dispersive channels. The performance of the MC-CDMA aided $\mathcal{G}_{2}-\operatorname{STBC}\left(N_{T}, N_{R}\right)=(2,2)$, BPSK and $\mathcal{G}_{2}-\operatorname{STBC}(2,2)$, QPSK is also shown as benchmarker.

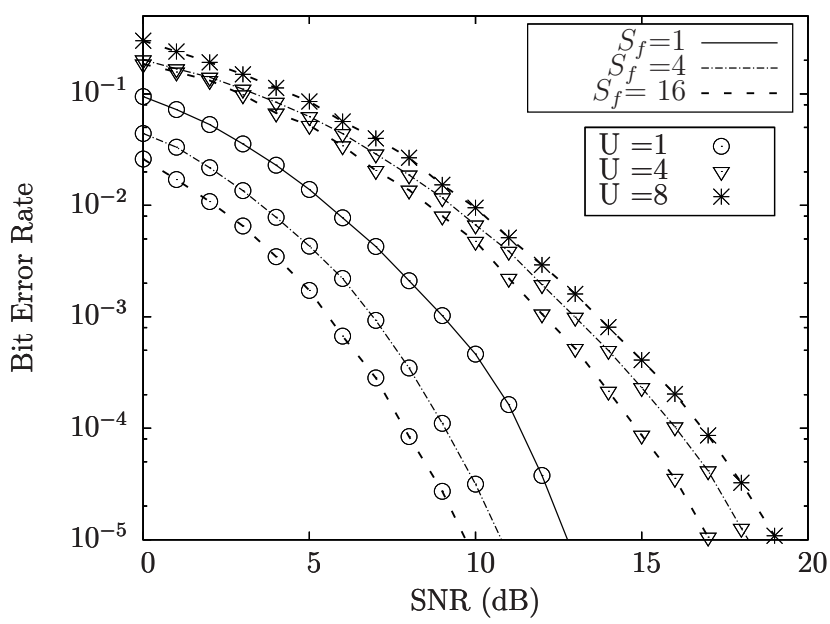

Fig. 7. BER performance of MC-CDMA aided STSK $(2,2,2,2,2)$ for the DL COST207-RA channel using different $S_{f}$ supporting $U$ users.

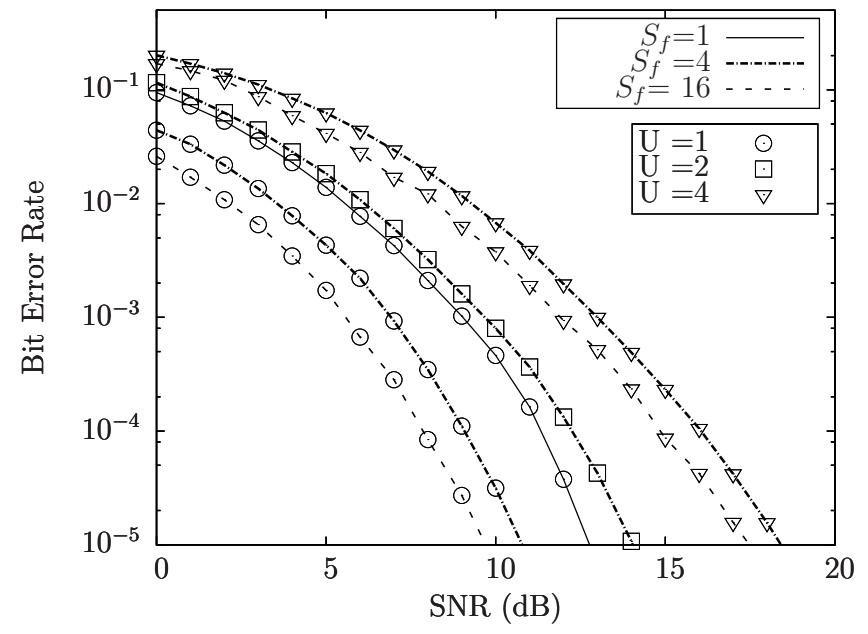

Fig. 8. Performance of MC-CDMA STSK $(2,2,2,2,2)$ UL for the COST207-RA channel using different $S_{f}$ supporting $U$ users. 


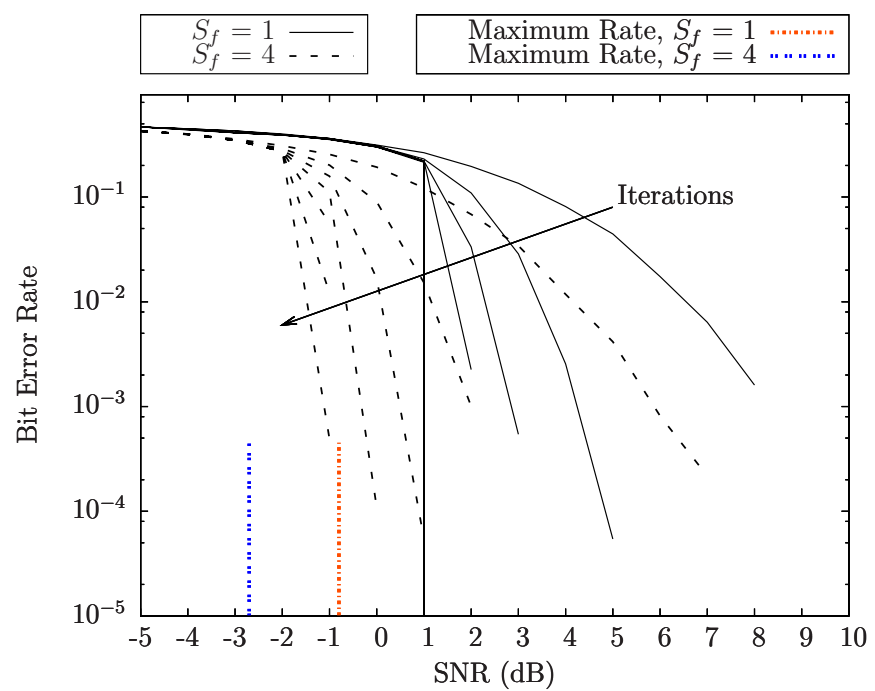

Fig. 9. BER of our channel-coded single-user MC-CDMA STSK $(2,2,2,4,4)$ system with $S_{f}=1$ and $S_{f}=4$ for the COST207-TU12 channel model of Table I. The maximum achievable rates with $S_{f}=1$ and $S_{f}=4$ are also shown as the ultimate benchmarker of the scheme.

BER after a few iterations. The maximum achievable rates, where the scheme can still exhibit an extremely low BER, were computed using EXIT chart analysis and are shown as the ultimate benchmarker of the proposed scheme. Specifically, as discussed in [8], [17], the area under the inner decoder's EXIT characteristic gives the maximum achievable rate for the specific scheme. The signal-to-noise ratios (SNRs), which provide maximum achievable rates for the MC-CDMA STSK $(2,2,2,4,4)$ schemes having spreading factors of $S_{f}=1$ and $S_{f}=4$ are computed and are shown in Fig. 9. The scheme is observed to exhibit an infinitesimally low BER, especially with higher value of $S_{f}$, after a few outer iterations.

\section{Conclusions}

In this contribution, both uncoded and channel-coded FD MC-CDMA aided STSK schemes are proposed. The proposed MC-CDMA aided STSK system is found to attain an improved performance, especially in dispersive multi-path channels, which are typical of high-rate urban scenarios. Moreover, the attainable performance improves upon increasing the spreading factor $S_{f}$, although as expected, it suffers from MUI under multiuser scenario.

The performance degradation of classic STSK system in broadband channels may be mitigated by orthogonal frequency division multiplexing (OFDM)-aided STSK system [9]. For further exploitation of the frequency diversity provided by FD spreading, while facilitating multi-user communications, we have employed MC-CDMA in our proposed scheme. The scheme has also the capability of benefitting from the employment of our joint single-stream ML-MUD.

The effectiveness of our system largely depends on the optimization of the DMs utilized. We have optimized the spreading matrices minimizing the pairwise symbol error probability by an exhaustive search so that the power constraint used in [7], [8] is satisfied. Furthermore, in order to reduce the computational complexity associated with the exhaustive search, genetic algorithm (GA)-aided DM optimization [18] may also be applied.

\section{ACKNOWLEDGMENT}

The authors would like to thank the Commonwealth Scholarship Commission in the UK for the financial support of this work. The financial support of the RC-UK under the auspices of the IU-ATC, of the EU under the Concerto project and of the European Research Council under its Advanced Fellow Grant is also gratefully acknowledged.

\section{REFERENCES}

[1] C. J. Foschini, "Layered space-time architecture for wireless communication in a fading environment when using multiple antennas," Bell Labs. Tech. J., vol. 1, no. 2, pp. 41-59, 1996.

[2] V. Tarokh, H. Jafarkhani, and A. Calderbank, "Space-time block codes from orthogonal designs," IEEE Trans. Inf. Theory, vol. 45, no. 5, pp. 1456-1467, Jul. 1999.

[3] B. Hassibi and B. M. Hochwald, "High-rate codes that are linear in space and time," IEEE Trans. Inf. Theory, vol. 48, no. 7, pp. 1804-1824, Jul. 2002.

[4] J. Heath, R.W. and A. Paulraj, "Linear dispersion codes for MIMO systems based on frame theory," IEEE Trans. Signal Process., vol. 50, no. 10, pp. 2429-2441, Oct. 2002.

[5] R. Mesleh, H. Haas, S. Sinanovic, C. W. Ahn, and S. Yun, "Spatial modulation," IEEE Trans. Veh. Technol., vol. 57, no. 4, pp. 2228-2241, Jul. 2008.

[6] J. Jeganathan, A. Ghrayeb, L. Szczecinski, and A. Ceron, "Space shift keying modulation for MIMO channels," IEEE Trans. Wireless Commun., vol. 8, no. 7, pp. 3692-3703, Jul. 2009.

[7] S. Sugiura, S. Chen, and L. Hanzo, "A universal space-time architecture for multiple-antenna aided systems," IEEE Commun. Surveys Tuts., vol. 14, no. 2, pp. 401-420, 2012.

[8] — "Coherent and differential space-time shift keying: A dispersion matrix approach," IEEE Trans. Commun., vol. 58, no. 11, pp. 32193230, Nov. 2010.

[9] M. Driusso, F. Babich, M. I. Kadir, and L. Hanzo, "OFDM aided spacetime shift keying for dispersive downlink channels," in Proc. IEEE VTC Fall, 2012, Sep. 2012, pp. 1-5.

[10] H. A. Ngo, C. Xu, S. Sugiura, and L. Hanzo, "Space-time-frequency shift keying for dispersive channels," IEEE Signal Process. Lett., vol. 18, no. 3, pp. 177-180, Mar. 2011.

[11] L. Hanzo, O. Alamri, M. El-Hajjar, and N. Wu, Near-Capacity MultiFunctional MIMO Systems (Sphere-Packing, Iterative Detection and Cooperation). New York, NY: John Wiley \& Sons, 2009.

[12] S. Hara and R. Prasad, "Overview of multi-carrier CDMA," IEEE Commun. Mag., vol. 35, no. 12, pp. 126-133, Dec. 1997.

[13] N. Yee, J. P. Linnartz, and G. Fettweis, "Multi-carrier CDMA in indoor wireless radio networks," in Proc. IEEE PIMRC'93, Sep. 1993, pp. 109113.

[14] L. Hanzo, M. Munster, B. J. Choi, and T. Keller, OFDM and MCCDMA for Broadcasting Multi-User Communications, WLANs and Broadcasting. New York, NY: John Wiley \& Sons, 2003.

[15] S. ten Brink, J. Speidel, and R.-H. Yan, "Iterative demapping and decoding for multilevel modulation," in Proc. IEEE GLOBECOM,1998, vol. 1, 1998, pp. 579-584 vol.1.

[16] J. Woodard and L. Hanzo, "Comparative study of turbo decoding techniques: an overview," IEEE Trans. Veh. Technol., vol. 49, no. 6, pp. 2208-2233, Nov. 2000.

[17] M. Tuchler, "Design of serially concatenated systems depending on the block length," IEEE Trans. Commun., vol. 52, no. 2, pp. 209-218, Feb. 2004.

[18] M. Jiang and L. Hanzo, "Unitary linear dispersion code design and optimization for MIMO communication systems," IEEE Signal Process. Lett., vol. 17, no. 5, pp. 497-500, May 2010. 